\title{
Diagnosis and Management of Pancreatic Adenocarcinoma in the Background of Chronic Pancreatitis: Core Issues
}

\author{
Narkhede Rajvilas A. ${ }^{a} \quad$ S. Desai Gunjan ${ }^{b}$ Prasad Pande P. ${ }^{c}$ Wagle Prasad K. ${ }^{d}$ \\ a Junior Consultant, Department of Gastrointestinal Surgery, Lilavati Hospital and Research Center, Mumbai, India; \\ ${ }^{b}$ Clinical Associate, Department of Gastrointestinal Surgery, Lilavati Hospital and Research Center, Mumbai, India; \\ 'Department of Gastrointestinal Surgery, DNB Gastrointestinal Surgery Third Year Resident, Lilavati Hospital and \\ Research Center, Mumbai, India; ${ }^{\mathrm{d} C}$ Consultant, Department of Gastrointestinal Surgery, Lilavati Hospital and Research \\ Center, Mumbai, India
}

\section{Keywords \\ Chronic pancreatitis - Pancreatic cancer · Pancreatic head mass · Pancreatic adenocarcinoma}

\begin{abstract}
Background: The incidence of pancreatic adenocarcinoma (PDAC) in patients with chronic pancreatitis (CP) is as high as $5 \%$. It is a commonly encountered diagnostic challenge in patients with CP on long-term follow-up. Summary: This review consolidates the existing literature on assessment of PDAC in background of $C P$, its evaluation through the available investigations, surgical management, and prognostication. Recent change in symptomatology of an otherwise stable CP should raise a suspicion of malignancy. Endoscopic ultrasound (EUS) is more specific and sensitive in establishing the diagnosis of PDAC compared to cross-sectional imaging (computed tomography/magnetic resonance imaging). Intraoperative assessment with careful palpation coupled with careful clinical judgment helps in differentiating between an inflammatory mass and pancreatic cancer. Confirmation can be obtained with either preoperative EUS-guided fine needle cytology/core biopsy or intraoperative core biopsy under ultrasound guidance. However, despite complete evaluation with above options, $1-6 \%$ patients often show malignancy
\end{abstract}

on final histopathological examination. Key Messages: Diagnosis of PDAC in CP needs a high index of suspicion. Crosssectional imaging has poor negative predictive value. CA 19-9 with MUC5AC combination may become an ideal investigation. EUS with core biopsy/frozen section has a good sensitivity and specificity and low false negative results. Complete radical resection should be the aim to get long-term survival.

C) 2019 S. Karger AG, Basel

\section{Introduction}

Chronic pancreatitis (CP) is a progressive inflammatory disease with irreversible functional and morphological changes in pancreas. $\mathrm{CP}$ is a major risk factor for development of pancreatic cancer, with a 2.3-18.5-fold increase in risk, and a head mass in CP must be investigated to rule out malignancy [1]. The presentation of $\mathrm{CP}$ - abdominal pain, steatorrhea, diabetes, weight loss, and obstructive jaundice, is very similar to pancreatic adenocarcinoma (PDAC).

There is a dearth of data on progression of CP into malignancy. Majority of the literature is derived from case-control or cohort studies, and historical cohorts

\section{KARGER}

(c) 2019 S. Karger AG, Basel

E-Mail karger@karger.com

www.karger.com/ddi
Prasad Wagle

Consultant, Department of Gastrointestinal Surgery

Lilavati Hospital and Research Center, Bandra West

Mumbai 400050 (India)

E-Maildrprasadwagle@gmail.com 
Table 1. Demographic differences in $\mathrm{CP}$ and pancreatic cancer

\begin{tabular}{|c|c|c|}
\hline Characteristic & $\mathrm{CP}$ & Pancreatic cancer \\
\hline \multicolumn{3}{|l|}{ Age at diagnosis, years, $\%$} \\
\hline$<40$ & 35 & 2 \\
\hline $40-59$ & 50 & 8 \\
\hline$\geq 60$ & 15 & 90 \\
\hline Gender, male:female ratio & $4: 1$ & $1: 1$ \\
\hline Race & Non caucasians $>$ caucasians & Non caucasians $>$ caucasians \\
\hline \multicolumn{3}{|l|}{ Risk factors } \\
\hline Smoking & Major cofactor & Doubles the risk \\
\hline Alcohol & Strongest lifestyle-related risk factor & Not a major risk factor \\
\hline Genetic & PRSS1, CFTR & Polygenic, BRCA2 strongest \\
\hline
\end{tabular}

Table 2. Relative risk and lifetime risk of developing pancreatic cancer based on etiology of CP [3, 23]

\begin{tabular}{lcc}
\hline Etiology of CP & Relative risk (folds) [3] & Lifetime risk, \% [3] \\
\hline Hereditary pancreatitis & 69 & $40-55$ \\
Tropical pancreatitis & $5-100$ & Not available \\
Others, unspecified & 13 & $2-4$ \\
\hline & HR (folds) [92] & \\
\hline Alcoholic ancreatitis & 1.72 & \\
Hereditary pancreatitis & 3.77 & \\
Hyperlipidemic CP & 0 & \\
Posttraumatic CP & 0 & \\
\hline \multicolumn{2}{c}{ CP, chronic pancreatitis; HR, hazard ratio. } &
\end{tabular}

have been used in some meta-analyses. The inciting factors responsible for this malignant transformation, and the population at high risk for it, have not been described. Early diagnosis of CP and referral for surgical intervention within 3 years of diagnosis, even in the absence of a head mass, is the only factor known to reduce the risk of malignant transformation [2]. However, further studies are needed to identify patients benefitting from early intervention and decrease the cancer risk. This review consolidates and elaborates the existing literature on approach to diagnosis of malignancy in $\mathrm{CP}$ and its management.

\section{Epidemiology}

The worldwide incidence of CP is $5-12 / 100,000$ population/year. This matches with the worldwide incidence of PDAC which is $6-12 / 100,000 /$ year [3]. Four to seven percent of patients with $\mathrm{CP}$ develop PDAC within 20 years of diagnosis and by age of 70 , nearly $40 \%$ of patients with hereditary pancreatitis will develop PDAC [1]. Table 1 elaborates the demographic differences in $\mathrm{CP}$ and PDAC.

The relative risk and lifetime risk of malignancy are highest for hereditary pancreatitis and tropical pancreatitis (Table 2), and presence of these diagnoses should prompt close surveillance to diagnose malignancy at an early stage.

\section{Clinical Suspicion of Malignancy in CP and Related Difficulties}

Diagnosis of CP peaks at 45-54 years [4], and PDAC is usually diagnosed in patients in their 7 th decade with median age of onset being 71 years [4]. The subtle changes in natural course of the disease, and appearance of a new symptom, should arouse suspicion regarding a ma- 
Table 3. Features suspicious for malignancy in CP [23]

Features suspicious for malignancy in $\mathrm{CP}$

Diagnosis of hereditary/tropical pancreatitis

Reappearance of pain after pain relief

Appearance of obstructive jaundice

Markedly dilated pancreatic duct on imaging

Unexplained weight loss despite pancreatic enzyme therapy

Markedly elevated CA 19-9

Pancreatic head mass on imaging

Vascular invasion on imaging

$\mathrm{CP}$, chronic pancreatitis.

lignant transformation and prompt investigations. These alarming signs and symptoms are elaborated in Table 3.

Subtle changes in symptomatology require a prompt and thorough evaluation. New onset diabetes or recent worsening of previously well-controlled diabetes, appearance of intractable pain, change in character of pain to a dull-aching continuous type, significant weight loss, and deep jaundice should alert the surgeon [3]. A palpable gall bladder is highly unusual in CP with jaundice and increases the suspicion of malignancy. The jaundice is deep (serum bilirubin $\geq 10 \mathrm{mg} / \mathrm{dL}$ ) in malignancy on background of $\mathrm{CP}$ owing to progressive and complete nature of obstruction in malignant biliary stricture [5]. The brief pathogenesis of PDAC from CP is described in Figure 1.

\section{Evaluation for Suspicious Malignancy in CP}

A malignancy in $\mathrm{CP}$ can be seen radiologically as a lesion in diffuse CP or mass forming focal CP [6-8]. An inflammatory lesion in head of pancreas, called a pseudotumor, can be seen in 10\% of presumed and resected adenocarcinomas [9]. Due to the radically different approaches to management of these 2 conditions, it is paramount to do appropriate, and often exhaustive investigations to exclude malignancy [10].

PDAC, often hypovascular, induces a florid desmoplastic reaction [11]. These 2 pathological aspects dictate its radiological findings $[12,13]$. Cross-sectional imaging such as transabdominal ultrasound (USG), contrast-enhanced computed tomography (CECT), or magnetic resonance imaging (MRI) should be performed for metastatic lesions, ascites, lymphadenopathy, vascular involvement, tumor characteristics, characteristics of nontumorous pancreas, and ductal findings (both main pancreatic duct [MPD] and common bile duct [CBD]) $[7,14]$.

Diagnosis and Management of PDAC in

the Background of CP: Core Issues
USG is seldom useful unless the patient has metastatic disease to liver and/or ascites, in which case USG-guided biopsy or fluid cytology can establish the diagnosis. Contrast-enhanced USG in arterial and venous phases has no added benefit, unless the malignancy is advanced with vascular involvement [15].

A triple-phase pancreas protocol CECT scan is usually the first investigation [7]. Normal pancreas shows postcontrast enhancement followed by washout [16]. PDAC is seen as a hypoenhancing lesion in pancreas with upstream pancreatic parenchymal atrophy in $80-82 \%$ cases [7]. This lesion enhancement is very gradual, progressive with a delayed peak in the portal phase or delayed scan at $4 \mathrm{~min}$ and usually has no washout [16]. This characteristic of tumor, if present, has sensitivity and specificity of 74 and $93 \%$, respectively [17]. Segmental pancreatic atrophy upstream of the lesion or in the body or tail of pancreas is also a suspicious finding [7].

PDAC is associated with double duct sign (dilated MPD and CBD with an abrupt cutoff at the mass) more commonly in head of pancreas mass than body mass $[7,8]$. Ductal dilatation in PDAC is significantly more than in CP. A study has shown that MPD dilatation $>11.5 \mathrm{~mm}$ and common bile duct dilatation $>14.5 \mathrm{~mm}$ is a specific marker of PDAC in CP [8]. In PDAC, the lesion does not contain any ducts, whereas a pseudotumor contains main duct or isolated dilated side branches within, known as the duct penetrating sign seen in $85 \%$ of CP and only $4 \%$ of PDAC [18]. Vascular sheathing at origin of celiac artery and superior mesenteric artery by a hypodense mass is suggestive of malignancy. However, vascular sheathing in an isodense mass that is also seen in type I autoimmune pancreatitis with mesenteritis and/or retroperitoneal fibrosis $[19,20]$.

Blood flow is the single most important parameter [6] in perfusion CT. Blood flow and blood volume as well as peak enhancement are 45 and $43 \%$ lower in PDAC, respectively, compared to CP. Also, time to peak enhancement is $15 \%$ higher for PDAC compared to CP. These parameters are useful when an isodense mass is found on CECT $[6,21]$.

T1 MRI images cannot differentiate pseudotumor from PDAC lesion. T2 images show a different signal intensity for tumor similar to that in CT [22]. Duct penetrating sign, double duct sign, and parenchymal atrophy can be seen. Additional MRI sequences are being studied for this scenario [10,23]. MR spectroscopy relies on lipid content of tissue. Lipid content is lower in CP due to fibrosis [24]. Diffusion MRI shows restricted diffusion in both lesions, with the restriction being more in PDAC. However, the overlap is significant and hence, it cannot be used to label the lesion conclusively [25]. 
Fig. 1. Pathogenesis of PDAC in background of CP. PDAC, pancreatic adenocarcinoma; $\mathrm{CP}$, chronic pancreatitis; DNA, deoxyribonucleic acid.

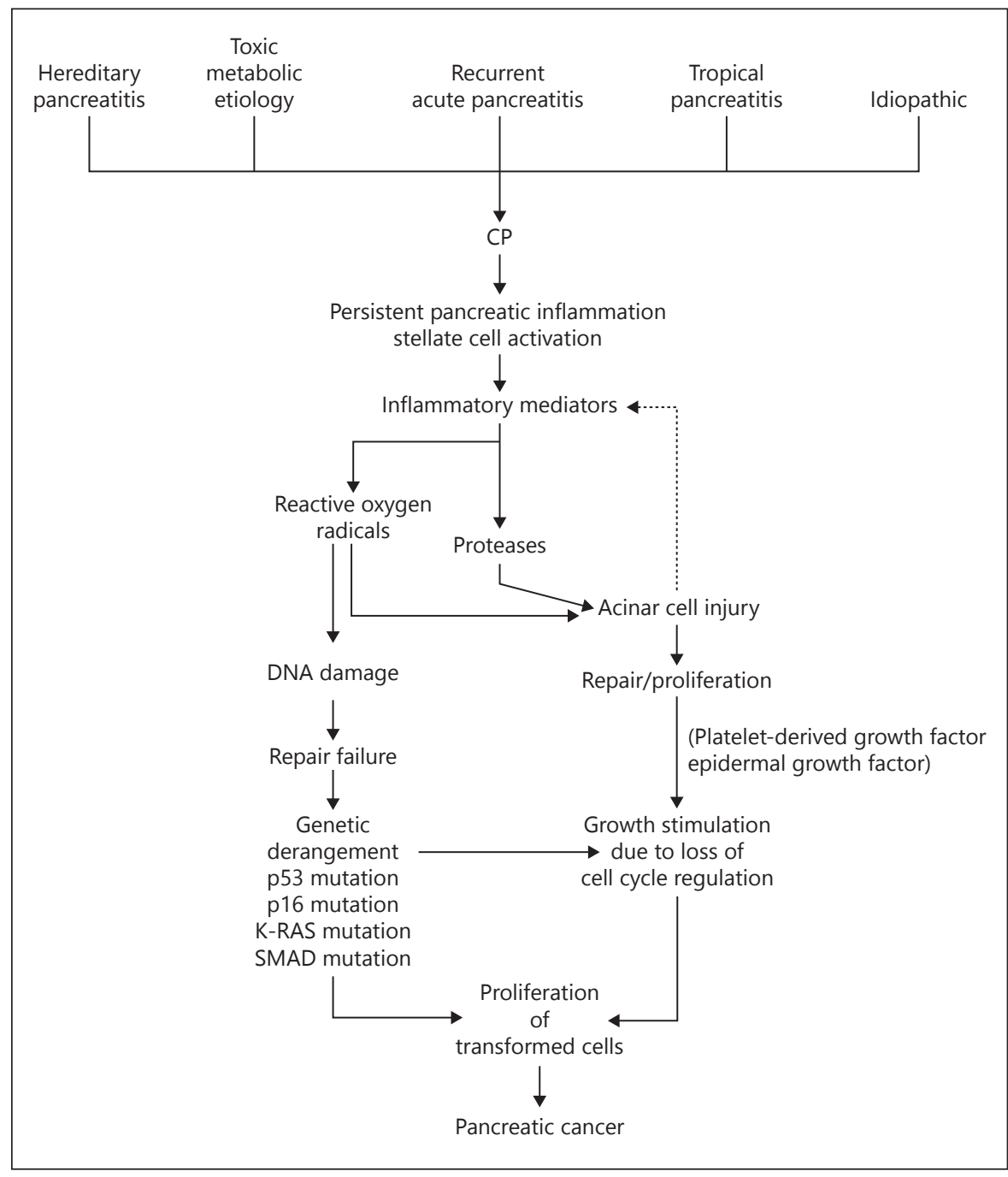

In gadolinium-enhanced gradient echo dynamic MRI, a nontumorous pancreas in patients with $\mathrm{CP}$ shows a gradual enhancement with a peak in delayed phase, similar to that of PDAC, again with a significant overlap [26].

To summarize MRI findings, hepatic metastasis, ascites, vascular involvement, ductal cut off, which can also be seen on CECT and USG, in the presence of a heterogeneous hypointense mass are suggestive of PDAC. Presence of cysts, calcifications, and a homogeneous mass is suggestive of CP $[10,23,26]$.

Endoscopic ultrasound (EUS) uses high-frequency probes in stomach to evaluate pancreas, overcoming the limitation of USG of obscured view due to intervening bowel gas [27]. Echotexture of the lesion as well as of the rest of the pancreas, its boundaries, ductal characteristics, lymphadenopathy, and vascular involvement can be studied better with EUS. Features favoring a pseudotumor include ho- mogeneous pattern with hyperechoic septations, calcifications, cysts, multilobular appearance, and positive Doppler signals in the lesion $[28,29]$. On the other hand, PDAC is suggested by hypoechoic mass, periductal hypoechogenecity, and displacement of calcifications [30]. The sensitivity and specificity for EUS alone are 64 and 75\%, respectively, for the detection of PDAC in CP [29]. However, in the presence of a pseudotumor, EUS alone cannot distinguish PDAC from mass forming CP [23].

Additional techniques used during EUS do not help in detection of a mass, but help to differentiate between pseudotumor and PDAC once detected, and include fine needle aspiration cytology/biopsy (FNAC/B), EUS elastography, contrast-enhanced EUS, and digital image analysis - a more objective computer-based method of image analysis [31-33]. EUS-FNAC has a very high sensitivity $(>85 \%)$ and specificity ( $>95 \%)$ for diagnosis of 
malignancy in a solid pancreatic mass $[33,34]$. However, this sensitivity and specificity decrease to $<75 \%$ on the background of CP $[35,36]$. This can be increased to $90 \%$ by $>7$ FNAC passes, using larger needle or by doing a core or suction biopsy followed by on site evaluation by an experienced cytopathologist [37-39].

EUS elastography measures stiffness of tissue. Benign tissue is more pliant compared to a malignant tumor. Nowadays, quantitative measures such as strain ratio can be used which is 18.12 for adenocarcinoma compared to 3.38 for pseudotumor and 1.68 for normal pancreas, which has sensitivity and specificity of $95 \%$ for this indication [ 40 , 41]. Contrast-enhanced EUS helps in evaluating the intravascular distribution of contrast. PDAC being hypovascular shows a delayed and decreased uptake of contrast compared to pseudotumor with sensitivity and specificity of 95\% to differentiate pseudotumor from PDAC [42-44].

Positron emission tomography-CT (PET-CT) is not routinely indicated. The clinical situations where the lesion is isodense/isointense on conventional imaging (CT/ MRI/EUS), when multiple large stones/calcifications obscure the EUS field, or when lesion has extensive cystic necrotic areas, are scenarios where PET-CT may be of value $[11,23]$. However, the fibrosis associated with PDAC often results in a hypometabolic lesion [45]. With CP, the flurodeoxyglucose uptake is diffuse and low grade, whereas the uptake is focal and high grade in PDAC [46]. Diagnostic yield can be increased by a targeted biopsy from the most hypermetabolic focus [47]. PET-CT is more sensitive than CECT for lesions up to $2 \mathrm{~cm}$ in size [48].

\section{Laboratory Investigations}

Serum bilirubin levels are seldom greater than $10 \mathrm{mg} / \mathrm{dL}$ in $\mathrm{CP}$, and the pattern is of a rise to peak followed by a fall as inflammation subsides [5]. In PDAC, bilirubin progressively increases till biliary decompression is done [49]. The sensitivity for suspecting malignancy was $100 \%$ when serum bilirubin $>5.8 \mathrm{mg} / \mathrm{dL}$, CBD $>14.5 \mathrm{~mm}, \mathrm{MPD}>11.5 \mathrm{~mm}$, and CA $19-9>127 \mathrm{U} / \mathrm{mL}$ were all present in a patient. However, this is very rarely seen in practice [8].

CA $19-9$ is the gold standard PDAC biomarker and is the single most important predictive biomarker of malignancy in mass forming $\mathrm{CP}$ with a sensitivity and specificity of 77 and $86 \%$, respectively [31]. However, it has a few limitations. Five to ten percent population who is Lewis blood group antigen negative do not express CA 19-9 [50, 51]. Also, value can be elevated in obstructive jaundice, cholan-

Diagnosis and Management of PDAC in

the Background of CP: Core Issues gitis, cirrhosis, and CP in absence of malignancy. Sensitivity for detection of early PDAC is $40-50 \%$ and specificity is $68-91 \%$ with a false positive rate of $23 \%[52,53]$.

Interleukin 8 , a proinflammatory marker of CXC chemokine family, was found to be more commonly expressed in PDAC (55.6\%) compared to para-cancer pancreatic tissue $(25.9 \%)$, and $\mathrm{CP}(0 \%)$ and was found to predict the prognosis of PDAC [54]. Combination of CA 19-9 with various other biomarkers has been studied, such as CA 19-9, albumin, and insulin-like growth factor-1, or CA 19-9 with C-reactive protein and interleukin 8, or CA 19-9 with carcinoembryonic antigen and tissue inhibitor of metalloproteinase, but none of them has been found to be diagnostic [55-57]. Carcinoembryonic antigen is not specific, is not produced by all pancreatic tumors, and is not as sensitive as CA 19-9 for early PDAC [58].

Various noninvasive biomarkers in saliva, feces, urine, and pancreatic juice have been studied [59]. Microribonucleic acids (miRNAs), noncoding RNAs that produce epigenetic changes in deoxyribonucleic acid, are often deregulated in pancreatic diseases. Quantitative real-time polymerase chain reaction, microarrays, and next-generation sequencing assays are used to measure miRNA 21, miRNA 155, and miRNA 210 levels in tissue, serum, and stool, respectively [60, 61]. Methylated biomarkers such as KRAS are also found to be $75 \%$ sensitive and 95\% specific for PDAC in comparison to $\mathrm{CP}$ and normal controls. Circulating tumor cells and exosomes have also been studied, but none of these, either alone or in combination, have been found to be superior to CA 19-9 [62]. Metabolic signature to separate PDAC from $\mathrm{CP}$ has been proposed that contains CA 19-9 along with 9 metabolites, that is, proline, phosphatidylcholine, 2 sphingomyelins, ceramide, pyruvate, histidine, isocitrate, and sphingosine-1-phosphate. It has been found to be $85 \%$ sensitive, $94.9 \%$ specific, and has a diagnostic accuracy of $90 \%$ to distinguish PDAC from CP. Its negative predictive value is $99.9 \%$, and false negative rate is only $11 \%[63]$.

The biomarker combination found to be very promising is MUC5AC with CA 19-9 [31]. MUC5AC is a mucin family member produced from pancreatic intraepithelial neoplasm type IA, IB and well differentiated as well as poorly differentiated PDAC. MUC5AC is undetectable in $\mathrm{CP}$, normal pancreas, and islet cell tumors [64, 65]. Its levels are also affected by elevated bilirubin levels. Tissue levels can be measured by immunohistochemistry, whereas serum levels can be measured by enzyme-linked immunosorbent assay. This combination outperforms both tests individually. A cutoff value of $20.4 \mathrm{ng} / \mathrm{mL}$ was found to 
differentiate all benign and normal controls from early PDAC [31]. PAM4 is a monoclonal antibody with high specificity for MUC1, which is found to be expressed in PDAC as compared to normal pancreatic tissue and pancreatitis [66]. PAM4 has also been recently found to have better sensitivity and specificity than CA 19-9 [67, 68]. Further studies may provide the key biomarker panel to detect PDAC in CP.

\section{Intraoperative Evaluation}

If malignancy is diagnosed or cannot be ruled out on complete evaluation, pancreaticoduodenectomy is the preferred choice for surgery [69]. In suspicious cases, a diagnostic laparoscopy might help to rule out metastatic disease missed at the preoperative evaluation [70].

Intraoperative ultrasound (IOUS) is often used; however, distinguishing malignancy from inflammatory head mass is difficult. Routine use of IOUS for pancreatic surgery was found to be helpful in $22.9 \%$ patients and guided surgical procedure in over $50 \%$ cases [71]. Obvious findings such as involvement of superior mesenteric vein, portal vein, splenic vein, or superior mesenteric artery confirm malignancy. A study showed high accuracy of IOUS in the assessment of suspicious cases of PDAC, and an addition of biopsy with histopathological examination did not improve its diagnostic value [72]. However, concerns of rapid intraabdominal spread of tumor cells have been raised following multiple intraoperative biopsies [72]. Also, negative or equivocal findings maintain the dilemma.

Earlier reports questioned the role of frozen sections due to its very high false negative results (70\%) [73] and associated low sensitivity. Diagnosis can be achieved by considering major criteria and minor criteria described by Hyland et al. [74] as shown in Table 4.

The accuracy of core biopsy was found to be superior to wedge biopsy (77-86 vs. 38-75\%) [75, 76]. Wedge biopsy, though under vision, often runs the risk of superficial biopsy because of the fear of a pancreatic ductal injury [77]. In a study by Fancellu et al. [78], complete excavation of pancreatic head in suspected patients with head mass can provide larger pancreatic tissue for frozen analysis and was shown to increase accuracy in identifying malignancy.

The accuracy of frozen section and histological interpretation has increased from 65 to $75 \%$ in 1980 s to above $90 \%$ in recent studies $[75,76,79]$. The sensitivity was found between 70.7 and $89.7 \%$ across various stud-
Table 4. Criteria on frozen section to identify PDAC [74]

\begin{tabular}{ll}
\hline Major criteria & Minor criteria \\
\hline $\begin{array}{l}\text { 1. Nuclear size variation of 4:1 } \\
\text { or greater between ductal } \\
\text { epithelial cells }\end{array}$ & $\begin{array}{l}\text { 1. Huge, irregular epithelial } \\
\text { nucleoli }\end{array}$ \\
$\begin{array}{l}\text { 2. Incomplete ductal lumen } \\
\text { and disorganized duct }\end{array}$ & $\begin{array}{l}\text { 2. Necrotic glandular debris } \\
\text { distribution }\end{array}$ \\
& $\begin{array}{l}\text { 4. Glands unaccompanied by } \\
\text { connective tissue stroma } \\
\text { within smooth muscle }\end{array}$ \\
& bundles \\
& 5. Perineural invasion \\
\hline
\end{tabular}

PDAC, pancreatic adenocarcinoma.

ies, whereas positive predictive value was found to be nearly $100 \%$ [79-81]. However, the major concern with frozen analysis is the relatively low negative predictive value $(50 \%)$ [79]. The false negativity of frozen examination was $1.2-30 \%$, and this rate increases in the presence of background CP because of the presence of reactive duct changes or atrophy [82]. Extended evaluation of snap-frozen analysis can be done for dedifferentiation markers, wherein protein expression of cluster of differentiation 97, cluster of differentiation 95, and Fas$\mathrm{L}$ can distinguish between the normal pancreatic parenchyma or pancreatitis from PDAC [83]. Despite all this, nearly $12 \%$ of the lesions are not diagnosed [76]. Hence, it gives immense importance to sound clinical judgment based on clinical features, preoperative evaluation, and surgeon's intraoperative assessment in the treatment of such patients.

Fancellu et al. [78] did pancreatic head excavation routinely for pancreatic head mass with suspicion of malignancy, and pancreaticoduodenectomy was offered to patients with malignancy on frozen section. Such patients had similar outcomes with those who underwent pancreaticoduodenectomy for clinico-radiologically suspected PDAC with CP. Mahesh et al. [69] also advocated pancreaticoduodenectomy for a positive frozen analysis of cored out tissue. On oncological grounds, such situations violate the oncological principles and may prove fatal in the outcomes. However, the study population in both these studies is small (15 with Fancellu et al. [78] and 3 with Mahesh et al. [69]).

A peculiar situation is when final histopathological examination shows adenocarcinoma after a negative frozen section analysis. Mahesh et al. [69] followed 3 patients with palliative chemotherapy (gemcitabine based) and offered total pancreatectomy for one patient. Total pan- 
createctomy had better overall survival than that of palliative chemotherapy (39 vs. 19 months) [69]. However, it is difficult to come to any conclusion with such limited evidence.

Intraoperative endocytoscopy can be helpful in confirming the diagnosis in the suspicious areas intraductally. In this procedure, local spraying of dyes such as methylene blue is done followed by examination with endocytoscope to look for typical surface histology of the diseased part under suspicion to diagnose PDAC in CP. However, opening the duct for endocytoscopy as done in a report by Rao et al. [75] will violate the oncological principles, hence the outcomes are debated.

\section{Prognosis}

Outcomes of a curative pancreaticoduodenectomy for a resectable PDAC with or without $\mathrm{CP}$ are similar when matched stage by stage. Postoperative pancreatic fistula rate is lower in CP with PDAC owing to firm texture of pancreatic tissues; however, Chu et al. [84] showed similar rates. The fibrosis around the portal vein, often leading to portal hypertension, may make the dissection difficult and lead to excessive intraoperative bleeding [85]. Total pancreatectomy can be an option for a subgroup of patients with PDAC in CP on the theoretical assumption of chronically inflamed pancreas being fertile soil for development of PDAC.

\section{Role of Screening and Preventive Measures}

No guidelines for screening for PDAC have been formulated yet. United States preventive task force recommended against screening of general population for PDAC. Even the use of screening in high-risk groups like hereditary pancreatitis is controversial [3]. Screening with imaging modalities exhibits limitations in settings of background CP and other high-risk groups like hereditary pancreatitis and is not routine [86].

EUS, despite being highly sensitive, is not reliable for screening due to significant false positive and false negative results. Moreover, ideal frequency of EUS examination for screening purpose and whether this screening improves outcome is questionable [87]. Role of serum markers such as androstanediol glucuronide, metabolomics, CA 19-9 alone or in combination with miR-16, mutational KRAS analysis, expression analysis of UHRF1,
ATP7A, and aldehyde oxidase 1 in FNA or biopsy sample is still under evaluation [3, 86, 88-91].

Owing to poor long-term outcomes of PDAC, preventive strategies seem logical. However, there are no concrete means for prevention. A multicenter study from Japan showed reduced incidence ( 0.7 vs. $5.1 \%, p=0.03$, hazard ratio 0.11 ) of PDAC after surgical treatment of $\mathrm{CP}$ where most patients underwent combined drainage with resectional procedure [2]. Since follow-up was only 5 years in this study, similar long-term studies might give more credible results.

\section{Conclusion}

Diagnosis of PDAC in CP needs a high index of suspicion, and every attempt should be made to prove malignancy preoperatively. Cross-sectional imaging with/ out functional imaging can differentiate malignancy from an inflammatory head mass; however, it has poor negative predictive value. CA 19-9 with MUC5AC combination may become an ideal investigation. EUS and IOUS can aid in the diagnosis of PDAC with an addition of FNA/core biopsy/frozen section having a good sensitivity and specificity and relatively lower false negative results. Intraoperative assessment with palpation and IOUS give the highest accuracy for intraoperative assessment of pancreatic head mass. An experienced surgeon's intraoperative palpatory findings and clinical judgement remain the final key in decision making. Complete radical resection should be the aim to get long-term survival in these cases.

\section{Acknowledgement}

The review is prepared by searching the following terms in Google scholar, Pubmed, and Pubmed central and exploring the related articles appearing in the search results: PDAC, pancreatic cancer, $C P$, pancreatic head mass, and malignancy in $\mathrm{CP}$.

\section{Disclosure Statement}

The authors have no conflicts of interest.

\section{Funds/Grants}

This research did not receive any specific grant from funding agencies in the public, commercial, or not-for-profit sectors. 


\section{References}

1 Kong X, Sun T, Kong F, Du Y, Li Z: Chronic pancreatitis and pancreatic cancer. Gastrointest Tumors 2014;1:123-134.

2 Ueda J, Tanaka M, Ohtsuka T, Tokunaga S, Shimosegawa T; Research Committee of Intractable Diseases of the Pancreas: Surgery for chronic pancreatitis decreases the risk for pancreatic cancer: a multicenter retrospective analysis. Surgery 2013;153:357-364.

3 Raimondi S, Lowenfels AB, Morselli-Labate AM, Maisonneuve P, Pezzilli R: Pancreatic cancer in chronic pancreatitis; aetiology, incidence, and early detection. Best Pract Res Clin Gastroenterol 2010;24:349-358.

4 Yadav D, Lowenfels AB: The epidemiology of pancreatitis and pancreatic cancer. Gastroenterology 2013;144:1252-1261.

5 Frey CF, Suzuki M, Isaji S: Treatment of chronic pancreatitis complicated by obstruction of the common bile duct or duodenum. World J Surg 1990;14:59-69.

6 Yadav AK, Sharma R, Kandasamy D, Pradhan RK, Garg PK, Bhalla AS, et al: Perfusion CT can it resolve the pancreatic carcinoma versus mass forming chronic pancreatitis conundrum? Pancreatology 2016;16:979-987.

7 Frampas E, Morla O, Regenet N, Eugène T, Dupas B, Meurette G: A solid pancreatic mass: tumour or inflammation? Diagn Interv Imaging 2013;94:741-755.

8 Perumal S, Palaniappan R, Pillai SA, Velayutham V, Sathyanesan J: Predictors of malignancy in chronic calcific pancreatitis with head mass. World J Gastrointest Surg 2013;5: 97-103.

9 Kajiwara M, Gotohda N, Konishi M, Nakagohri T, Takahashi S, Kojima M, et al: Incidence of the focal type of autoimmune pancreatitis in chronic pancreatitis suspected to be pancreatic carcinoma: experience of a single tertiary cancer center. Scand J Gastroenterol 2008;43:110-116.

10 Dutta AK, Chacko A: Head mass in chronic pancreatitis: inflammatory or malignant. World J Gastrointest Endosc 2015;7:258-264.

11 Jha P, Bijan B: PET/CT for pancreatic malignancy: potential and pitfalls. J Nucl Med Technol 2015;43:92-97.

12 Bachem MG, Schünemann M, Ramadani M, Siech M, Beger H, Buck A, et al: Pancreatic carcinoma cells induce fibrosis by stimulating proliferation and matrix synthesis of stellate cells. Gastroenterology 2005;128: 907-921.

13 Erkan M, Reiser-Erkan C, Michalski CW, Deucker S, Sauliunaite D, Streit S, et al: Cancer-stellate cell interactions perpetuate the hypoxia-fibrosis cycle in pancreatic ductal adenocarcinoma. Neoplasia 2009;11: 497-508.

14 Hocke M, Schulze E, Gottschalk P, Topalidis T, Dietrich CF: Contrast-enhanced endoscopic ultrasound in discrimination between focal pancreatitis and pancreatic cancer. World J Gastroenterol 2006;12:246-250.
15 Dietrich CF, Ignee A, Braden B, Barreiros AP, Ott M, Hocke M: Improved differentiation of pancreatic tumors using contrast-enhanced endoscopic ultrasound. Clin Gastroenterol Hepatol 2008;6:590-597.e1.

16 Yamada Y, Mori H, Matsumoto S, Kiyosue H, Hori Y, Hongo N: Pancreatic adenocarcinoma versus chronic pancreatitis: differentiation with triple-phase helical CT. Abdom Imaging 2010;35:163-171.

17 DelMaschio A, Vanzulli A, Sironi S, Castrucci M, Mellone R, Staudacher C, et al: Pancreatic cancer versus chronic pancreatitis: diagnosis with CA19-9 assessment, US, CT, and CT-guided fine-needle biopsy. Radiology 1991;178:95-99.

18 Ichikawa T, Sou H, Araki T, Arbab AS, Yoshikawa T, Ishigame K, et al: Duct-penetrating sign at MRCP: usefulness for differentiating inflammatory pancreatic mass from pancreatic carcinomas. Radiology 2001;221:107-116.

19 Ohara H, Nakazawa T, Sano H, Ando T, Okamoto T, Takada H, et al: Systemic extrapancreatic lesions associated with autoimmune pancreatitis. Pancreas 2005;31:232-237.

20 Vlachou PA, Khalili K, Jang HJ, Fischer S, Hirschfield GM, Kim TK: IgG4-related sclerosing disease: autoimmune pancreatitis and extrapancreatic manifestations. RadioGraphics 2011;31:1379-1402.

21 Yin Q, Zou X, Zai X, Wu Z, Wu Q, Jiang X, et al: Pancreatic ductal adenocarcinoma and chronic mass-forming pancreatitis: differentiation with dual-energy MDCT in spectral imaging mode. Eur J Radiol 2015;84:24702476.

22 Ragozzino A, Scaglione M: Pancreatic head mass: what can be done? Diagnosis: magnetic resonance imaging. JOP 2000;1(3 suppl): 100-107.

23 Pongprasobchai S, Kongkam P, Rerknimitr R: Pancreatic disorders \& therapy surveillance of pancreatic ductal adenocarcinoma in chronic pancreatitis? An Ongoing Challenge 2015;5: $1-5$.

24 Cho SG, Lee DH, Lee KY, Ji H, Lee KH, Ros $\mathrm{PR}$, et al: Differentiation of chronic focal pancreatitis from pancreatic carcinoma by in vivo proton magnetic resonance spectroscopy. J Comput Assist Tomogr 2005;29:163169.

25 Huang WC, Sheng J, Chen SY, Lu JP: Differentiation between pancreatic carcinoma and mass-forming chronic pancreatitis: usefulness of high b value diffusion-weighted imaging. J Dig Dis 2011;12:401-408.

26 Johnson PT, Outwater EK: pancreatic carcinoma versus chronic pancreatitis: dynamic MR imaging. Radiology 1999;213-218.

27 Irisawa A, Sato A, Sato M, Ikeda T, Suzuki R, Ohira H: Early diagnosis of small pancreatic cancer: role of endoscopic ultrasonography. Dig Endosc 2009;21(suppl 1):S92-S96.

28 Holt BA, Varadarajulu S: Features of chronic pancreatitis and associated masses: a focus on endosonography. Video J Encycl GI Endosc 2014;2:50-54.

29 Ardengh JC, Lopes CV, Campos AD, Pereira de Lima LF, Venco F, Módena JL: Endoscopic ultrasound and fine needle aspiration in chronic pancreatitis: differential diagnosis between pseudotumoral masses and pancreatic cancer. JOP 20078:413-421.

30 Lee SH, Ozden N, Pawa R, Hwangbo Y, Pleskow DK, Chuttani R, et al: Periductal hypoechoic sign: an endosonographic finding associated with pancreatic malignancy. Gastrointest Endosc 2010;71:249-255.

31 Kaur S, Smith LM, Patel A, Menning M, Watley DC, Malik SS, et al: A combination of MUC5AC and CA19-9 improves the diagnosis of pancreatic cancer: a multicenter study. Am J Gastroenterol 2017;112:172-183.

32 Mei M, Ni J, Liu D, Jin P, Sun L: EUS elastography for diagnosis of solid pancreatic masses: a meta-analysis. Gastrointest Endosc 2013; 77:578-589.

33 Rijkers AP, Valkema R, Duivenvoorden HJ, van Eijck CH: Usefulness of F-18-fluorodeoxyglucose positron emission tomography to confirm suspected pancreatic cancer: a metaanalysis. Eur J Surg Oncol 2014;40:794-804.

34 Fritscher-Ravens A, Brand L, Knöfel WT, Bobrowski C, Topalidis T, Thonke F, et al: Comparison of endoscopic ultrasound-guided fine needle aspiration for focal pancreatic lesions in patients with normal parenchyma and chronic pancreatitis. Am J Gastroenterol 2002;97:2768-2775.

35 Varadarajulu S, Tamhane A, Eloubeidi MA: Yield of EUS-guided FNA of pancreatic masses in the presence or the absence of chronic pancreatitis. Gastrointest Endosc 2005;62: 728-736.

36 Iordache S, Săftoiu A, Cazacu S, Gheonea DI, Dumitrescu D, Popescu C, et al: Endoscopic ultrasound approach of pancreatic cancer in chronic pancreatitis patients in a tertiary referral centre. J Gastrointestin Liver Dis 2008; 17:279-284.

37 Bang JY, Varadarajulu S: Neoplasia in chronic pancreatitis: how to maximize the yield of endoscopic ultrasound-guided fine needle aspiration. Clin Endosc 2014;47:420-424.

38 Hewitt MJ, McPhail MJ, Possamai L, Dhar A, Vlavianos P, Monahan KJ: EUS-guided FNA for diagnosis of solid pancreatic neoplasms: a meta-analysis. Gastrointest Endosc 2012;75: 319-331.

39 LeBlanc JK, Ciaccia D, Al-Assi MT, McGrath $\mathrm{K}$, Imperiale T, Tao LC, et al: Optimal number of EUS-guided fine needle passes needed to obtain a correct diagnosis. Gastrointest Endosc 2004;59:475-481.

40 Rijkers AP, Valkema R, Duivenvoorden HJ, van Eijck CHJ: Usefulness of F-18-fluorodeoxyglucose positron emission tomography to confirm suspected pancreatic cancer: a meta-analysis. Eur J Surg Oncol 2014;40: 794-804. 
41 Iglesias-Garcia J, Larino-Noia J, Abdulkader I, Forteza J, Dominguez-Munoz JE: Quantitative endoscopic ultrasound elastography: an accurate method for the differentiation of solid pancreatic masses. Gastroenterology 2010; 139:1172-1180.

42 Gong T, Hu DM, Zhu Q: Contrast-enhanced EUS for differential diagnosis of pancreatic mass lesions: a meta-analysis. Gastrointest Endosc 2012;76:301-309.

43 Săftoiu A, Iordache SA, Gheonea DI, Popescu C, Maloş A, Gorunescu F, et al: Combined contrast-enhanced power Doppler and realtime sonoelastography performed during EUS, used in the differential diagnosis of focal pancreatic masses (with videos). Gastrointest Endosc 2010;72:739-747.

44 Seicean A, Badea R, Stan-Iuga R, Mocan T, Gulei I, Pascu O: Quantitative contrast-enhanced harmonic endoscopic ultrasonography for the discrimination of solid pancreatic masses. Ultraschall Med 201015;31:571576.

45 Sahani DV, Bonaffini PA, Catalano OA, Guimaraes AR, Blake MA: State-of-the-art PET/ $\mathrm{CT}$ of the pancreas: current role and emerging indications. Radiographics 2012;32:11331158; discussion 1158-1160.

46 van Kouwen MC, Jansen JB, van Goor H, de Castro S, Oyen WJ, Drenth JP: FDG-PET is able to detect pancreatic carcinoma in chronic pancreatitis. Eur J Nucl Med Mol Imaging 2005;32:399-404.

47 Tatli S, Gerbaudo VH, Mamede M, Tuncali K, Shyn PB, Silverman SG: Abdominal masses sampled at PET/CT-guided percutaneous biopsy: initial experience with registration of prior PET/CT images. Radiology 2010;256: 305-311.

48 Delbeke D, Rose DM, Chapman WC, Pinson CW, Wright JK, Beauchamp RD, et al: Optimal interpretation of FDG PET in the diagnosis, staging and management of pancreatic carcinoma. J Nucl Med 1999;40:1784-1791.

49 Wapnick S, Hadas N, Purow E, Grosberg SJ: Mass in the head of the pancreas in cholestatic jaundice: carcinoma or pancreatitis? Ann Surg 1979;190:587-591.

50 Goggins M: Molecular markers of early pancreatic cancer. J Clin Oncol 2005;23:45244531.

51 Brody JR, Witkiewicz AK, Yeo CJ: The past, present, and future of biomarkers: a need for molecular beacons for the clinical management of pancreatic cancer. Adv Surg 2011;45: 301-321.

52 Kaur S, Baine MJ, Jain M, Sasson AR, Batra SK: Early diagnosis of pancreatic cancer: challenges and new developments. Biomark Med 2012;6:597-612.

53 Locker GY, Hamilton S, Harris J, Jessup JM, Kemeny N, Macdonald JS, et al: ASCO 2006 update of recommendations for the use of tumor markers in gastrointestinal cancer. J Clin Oncol 2006;24:5313-5327.

54 Chen Y, Shi M, Yu GZ, Qin XR, Jin G, Chen $\mathrm{P}$, et al: Interleukin-8, a promising predictor for prognosis of pancreatic cancer. World J Gastroenterol 2012;18:1123-1129.

55 Zhang P, Zou M, Wen X, Gu F, Li J, Liu G, et al: Development of serum parameters panels for the early detection of pancreatic cancer. Int J Cancer 2014;134:2646-2655.

56 Ferri MJ, Saez M, Figueras J, Fort E, Sabat M, López-Ben S, et al: Improved pancreatic adenocarcinoma diagnosis in jaundiced and nonjaundiced pancreatic adenocarcinoma patients through the combination of routine clinical markers associated to pancreatic adenocarcinoma pathophysiology. PLoS One. 2016;11:e0147214.

57 Brand RE, Nolen BM, Zeh HJ, Allen PJ, Eloubeidi MA, Goldberg M, et al: Serum biomarker panels for the detection of pancreatic cancer. Clin Cancer Res 2011;17:805-816.

58 Duraker N, Hot S, Polat Y, Höbek A, Gençler N, Urhan N: CEA, CA19-9, and CA 125 in the differential diagnosis of benign and malignant pancreatic diseases with or without jaundice. J Surg Oncol 2007;95:142-147.

59 Herreros-Villanueva M, Bujanda L: Non-invasive biomarkers in pancreatic cancer diagnosis: what we need versus what we have. Ann Transl Med 2016;4:134.

60 Li A, Yu J, Kim H, Wolfgang CL, Canto MI, Hruban RH, et al: MicroRNA array analysis finds elevated serum miR-1290 accurately distinguishes patients with low-stage pancreatic cancer from healthy and disease controls. Clin Cancer Res 2013;19:3600-3610.

61 Schultz NA, Dehlendorff C, Jensen BV, Bjerregaard JK, Nielsen KR, Bojesen SE, et al: MicroRNA biomarkers in whole blood for detection of pancreatic cancer. JAMA 2014;311: 392-404.

62 Matsubayashi H, Canto M, Sato N, Klein A, Abe T, Yamashita K, et al: DNA methylation alterations in the pancreatic juice of patients with suspected pancreatic disease. Cancer Res 2006;66:1208-1217.

63 Mayerle J, Kalthoff H, Reszka R, Kamlage B, Peter E, Schniewind B, et al: Metabolic biomarker signature to differentiate pancreatic ductal adenocarcinoma from chronic pancreatitis. Gut 2018;67:128-137.

64 Kaur S, Kumar S, Momi N, Sasson AR, Batra SK: Mucins in pancreatic cancer and its microenvironment. Nat Rev Gastroenterol Hepatol 2013;10:607-620.

65 Iacobuzio-Donahue CA, Ashfaq R, Maitra A, Adsay NV, Shen-Ong GL, Berg K, et al: Highly expressed genes in pancreatic ductal adenocarcinomas: a comprehensive characterization and comparison of the transcription profiles obtained from three major technologies. Cancer Res 2003;63:8614-8622.

66 Gold DV, Karanjawala Z, Modrak DE, Goldenberg DM, Hruban RH: PAM4-reactive MUC1 is a biomarker for early pancreatic adenocarcinoma. Clin Cancer Res 2007;13: 7380-7387.

67 Gold DV, Modrak DE, Ying Z, Cardillo TM, Sharkey RM, Goldenberg DM: New MUC1 serum immunoassay differentiates pancreatic cancer from pancreatitis. J Clin Oncol 2006; 24:252-258.

68 Ballehaninna UK, Chamberlain RS: The clinical utility of serum CA19-9 in the diagnosis, prognosis and management of pancreatic adenocarcinoma: an evidence based appraisal. J Gastrointest Oncol 2012;3:105-119.

69 Mahesh S, Lekha V, Manipadam JM, Venugopal A, Ramesh H: What can be done when the cored specimen in a Frey procedure for chronic pancreatitis is reported as adenocarcinoma? Indian J Gastroenterol 2016;35:486-488.

70 Taylor B: Carcinoma of the head of the pancreas versus chronic pancreatitis: diagnostic dilemma with significant consequences. World J Surg 2003;27:1249-1257.

71 Kaczmarek B: [The value of intraoperative ultrasonography during surgery for pancreatitis related changes of the pancreas]. Ann Acad Med Stetin 2000;46:123-136.

72 Brimienè V, Brimas G, Strupas K: Differential diagnosis between chronic pancreatitis and pancreatic cancer: a prospective study of 156 patients. Medicina (Kaunas) 2011;47:154162.

73 Harris PL, Rumley TO, Lineaweaver WC, Copeland EM: Pancreatic cancer: unreliability of frozen section in diagnosis. South Med J 1985; 78:1053-1056.

74 Hyland C, Kheir SM, Kashlan MB: Frozen section diagnosis of pancreatic carcinoma: a prospective study of 64 biopsies. Am J Surg Pathol 1981;5:179-191.

75 Rao G, Pradeep R, Mansard MJ, Ramji C, Banerjee R, Nageshwar Reddy D: Endocytoscopy assists in the intraoperative diagnosis of carcinoma in a patient with chronic pancreatitis. Endoscopy 2007;39(suppl 1):E317-E318.

76 Buice WS, Walker LG: The role of intra-operative biopsy in the treatment of resectable neoplasms of the pancreas and periampullary region. Am Surg 1989;55:307-310.

77 Lee YT: Tissue diagnosis for carcinoma of the pancreas and periampullary structures. Cancer 1982;49:1035-1039.

78 Fancellu A, Ginesu GC, Feo CF, Cossu ML, Puledda M, Pinna A, et al: Pancreatic head excavation for tissue diagnosis may reduce unnecessary pancreaticoduodenectomies in the setting of chronic pancreatitis. Hepatobiliary Pancreat Dis Int 2017;16:315-322.

79 Nelson DW, Blanchard TH, Causey MW, Homann JF, Brown TA: Examining the accuracy and clinical usefulness of intraoperative frozen section analysis in the management of pancreatic lesions. Am J Surg 2013;205:613617

80 Garcea G, Metcalfe MS, Berry DP, Robertson GS, Lloyd DM, Dennison AR: Is intraoperative confirmation of malignancy during pancreaticoduodenectomy mandatory? J Gastrointest Surg 2012;16:370-375.

81 Shyr YM, Su CH, Wu CW, Lui WY: Is pancreaticoduodenectomy justified for chronic pancreatitis masquerading as periampullary tumor? Hepatogastroenterology 2003;50:11631166.
Diagnosis and Management of PDAC in the Background of CP: Core Issues
Dig Dis 2019;37:315-324 DOI: $10.1159 / 000496507$ 
82 Cioc AM, Ellison EC, Proca DM, Lucas JG, Frankel WL: Frozen section diagnosis of pancreatic lesions. Arch Pathol Lab Med 2002; 126:1169-1173.

83 Boltze C, Schneider-Stock R, Aust G, Mawrin C, Dralle H, Roessner A, et al: CD97, CD95 and Fas-L clearly discriminate between chronic pancreatitis and pancreatic ductal adenocarcinoma in perioperative evaluation of cryocut sections. Pathol Int 2002;52:83-88.

84 Chu CK, Sarmiento JM, Park J, Staley CA, Galloway JR, Adsay NV, et al: Differences in presentation and perioperative outcome after pancreaticoduodenectomy for cancer and benign pancreatitis. Am Surg 2010;76:606-613.

85 Bachmann K, Izbicki JR, Yekebas EF: Chronic pancreatitis: modern surgical management. Langenbecks Arch Surg 2011;396:139-149.
86 Ulrich CD; Consensus Committees of the European Registry of Hereditary Pancreatic Diseases, Midwest Multi-Center Pancreatic Study Group, International Association of Pancreatology: Pancreatic cancer in hereditary pancreatitis: consensus guidelines for prevention, screening and treatment. Pancreatology 2001;1:416-422.

87 Poley JW, Kluijt I, Gouma DJ, Harinck F, Wagner A, Aalfs C, et al: The yield of first-time endoscopic ultrasonography in screening individuals at a high risk of developing pancreatic cancer. Am J Gastroenterol 2009;104:2175-2181.

88 Crnogorac-Jurcevic T, Gangeswaran R, Bhakta V, Capurso G, Lattimore S, Akada M, et al: Proteomic analysis of chronic pancreatitis and pancreatic adenocarcinoma. Gastroenterology 2005;129:1454-1463.
89 Jansa R, Prezelj J, Kocijancic A, Osredkar J, Ferlic F: Androstanediol glucuronide in patients with pancreatic cancer and in those with chronic pancreatitis. Horm Metab Res 1996;28:381-383.

90 Kobayashi T, Nishiumi S, Ikeda A, Yoshie T, Sakai A, Matsubara A, et al: A novel serum metabolomics-based diagnostic approach to pancreatic cancer. Cancer Epidemiol Biomarkers Prev 2013;22:571-579.

91 Gao L, He SB, Li DC: Effects of miR-16 plus CA19-9 detections on pancreatic cancer diagnostic performance. Clin Lab 2014;60:73-77.

92 Hao L, Zeng XP, Xin L, Wang D, Pan J, Bi YW, et al: Incidence of and risk factors for pancreatic cancer in chronic pancreatitis: a cohort of 1656 patients. Dig Liver Dis 2017;49:12491256. 